\title{
Dispositivo EROS en el manejo de la anorgasmia femenina: Estudio prospectivo de serie de casos en mujeres del Quindío
}

\author{
EROS device in the management of female anorgasmia: Prospective study of the series of cases in women in \\ Quindío
}

Franklin José Espitia-De La Hoz'** orcid.org/0000-0002-4581-9680

1 Clínica Sexológica, Armenia, Quindío, Colombia.

Fecha de recepción: Mayo 7 - $2018 \quad$ Fecha de revisión: Octubre $23-2018 \quad$ Fecha de aceptación: Diciembre 11 - 2018

Espitia- De La Hoz FJ. Dispositivo EROS en el manejo de la anorgasmia femenina: Estudio prospectivo de serie de casos en mujeres del Quindío. Univ. Salud. 2019;21(1):38-47. DOI: http://dx.doi.org/10.22267/rus.192101.138

\section{Resumen}

Introducción: La anorgasmia es el retraso persistente, o recurrente o ausencia de orgasmo después de una fase de excitación sexual normal, resultando en dificultades personales o interpersonales. El tratamiento se aborda desde diferentes perspectivas. Objetivo: Presentar los resultados de la efectividad y seguridad del uso del dispositivo EROS-CTD en el manejo de la anorgasmia femenina, en un grupo de mujeres del Quindío. Materiales y métodos: Estudio de reporte de casos, de tipo observacional, descriptivo, prospectivo, de mujeres intervenidas por trastorno del orgasmo, en Armenia, Quindío, Colombia, en el periodo de 2012 a 2017. Resultados: Se intervino 39 mujeres; edad media de 35,1 (DS $\pm 3,7$ ) años. La anorgasmia primaria fue del $61,53 \%$ y la secundaria del 38,36\%. El promedio de seguimiento del uso del dispositivo EROS-CTD, fue de 23,7 (DS $\pm 4,5$ ) meses por paciente. Al finalizar el estudio, la satisfacción global con el uso del dispositivo es del 89,74\%, ninguna de las mujeres presentó complicaciones relacionadas con el uso del aparato. Conclusiones: La efectividad del tratamiento de la anorgasmia femenina con el dispositivo EROS-CTD, ha beneficiado favorablemente a las mujeres del estudio.

Palabras clave: Efectividad; mujeres; orgasmo; seguridad; terapia. (Fuente: DeCS, Bireme).

\begin{abstract}
Introduction: Anorgasmia is the persistent, or recurrent, or absence of orgasm after a normal phase of sexual arousal, resulting in personal or interpersonal difficulties. Treatment is approached from different perspectives. Objective: To present the results of the effectiveness and safety of the use of the EROS-CTD in the management of female anorgasmia in a group of women from Quindío. Materials and methods: A descriptive, prospective observational study of case reports was carried out with women intervened by orgasm disorder in Armenia, Quindío, Colombia, from 2012 to 2017. Results: 39 women were intervened whose average age was 35.1 (DS \pm 3.7 ). Primary anorgasmia was $61.53 \%$ and the secondary was $38.36 \%$. The average follow-up of the use of EROS-CTD was 23.7 (DS \pm 4.5 ) months per patient. At the end of the study, overall satisfaction with the use of the device is $89.74 \%$, none of the women presented complications related to the use of the appliance. Conclusions: The effectiveness of the treatment of female anorgasmia with EROS-CTD has benefited the women of the study.
\end{abstract}

Key words: Effectiveness; women; orgasm; safety; therapy. (Source: DeCS, Bireme).

*Autor de correspondencia

Franklin José Espitia De La Hoz

e-mail: espitiafranklin@hotmail.com 


\section{Introducción}

El orgasmo femenino se interpreta como una sensación de felicidad y satisfacción después de la estimulación sexual adecuada, el cual se acompaña de contracciones rítmicas del útero, el ano y los músculos del piso pélvico, e igualmente es entendido como un estado de placer intenso a corto plazo ${ }^{(1-3)}$. La disfunción orgásmica se presenta cuando el orgasmo no ocurre o está notablemente retrasado ${ }^{(4)}$.

La anorgasmia femenina hace referencia a la incapacidad de la mujer para alcanzar el orgasmo luego de una estimulación sexual efectiva, es decir, que estando adecuadamente excitada se bloquea la respuesta orgásmica(2,4,5). Otra forma de definirla es el retraso persistente, o recurrente, o ausencia de orgasmo después de una fase de excitación sexual normal, lo que resulta en dificultades personales 0 interpersonales ${ }^{(6)}$.

La literatura reciente indica que alrededor de un $40 \%$ a $59 \%$ de las mujeres experimentan al menos una disfunción sexual a lo largo de su vida(7-9); hecho que puede estar asociado a la edad, educación, ocupación, consumo de fármacos, factores psicológicos, ambientales, culturales, creencias religiosas, trastornos psicológicos, cirugías ginecológicas y alteraciones fisiológicas (hormonales, vasculares, neurológicas y anatómicas), etc.(8,10,11).

La anorgasmia primaria está presente en un 5\% a $28,5 \%$ de las mujeres $(7,8,12)$, mientras que la secundaria oscila entre el 6,8\% y 59,09\%(7,8,13,14). Los problemas sexuales suelen causar dificultades en la relación de pareja; recientes estudios manifiestan que el $67 \%$ de los divorcios están relacionados con trastornos sexuales(12), y la anorgasmia no es la excepción(2,5).

El clítoris y la vagina son los sitios habituales de la estimulación sexual en la mujer para lograr el orgasmo; sin embargo, la estimulación periuretral, de las mamas y el pezón, así como las imágenes eróticas mentales $\mathrm{o}$ las fantasías, inducen e influyen en la aparición del

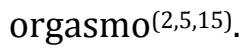

El clítoris tiene un papel significativo protagónico en la respuesta erógena primaria de la mujer, y dado su origen embriológico, anatomía y fisiología, la relevancia en el orgasmo femenino es incuestionable $(2,5,15,16)$. La explicación de todo esto es posible debido a que las ramas nerviosas del pudendo (nervio cavernoso, periférico y dorsal del clítoris), transmiten la estimulación sensorial que influye en la vasocongestión del área clitoral durante la excitación sexual(2,15-17), facilitando la respuesta orgásmica.

El tratamiento de la anorgasmia ha sido abordado desde diferentes perspectivas: psicoanalítica, farmacológica, cognitivoconductual $(3,4,18)$, entre otras. Las tasas de éxito, después de la terapia, oscilan el rango del 90\% para lograr el orgasmo durante la masturbación y el $75 \%$ para lograrlo en pareja(1,19-21). La terapia cognitivo-conductual para la anorgasmia se centra en promover cambios en las actitudes y pensamientos sexualmente relevantes, disminuyendo la ansiedad, aumentando la capacidad y satisfacción orgásmica. Los ejercicios de comportamiento tradicionalmente prescritos para inducir estos cambios incluyen masturbación dirigida, foco sensitivo y desensibilización sistemática. La educación sexual, la capacitación en habilidades de comunicación y los ejercicios de Kegel también se incluyen a menudo en programas de tratamiento cognitivo-conductual para la anorgasmia(22,23).

EROS clitoral therapy device (EROS-CTD), es un pequeño dispositivo médico de mano, hecho de suave plástico, del tipo vacuo vibrador, el cual tiene forma de ratón de computadora, trae inserto un capuchón del tamaño adecuado para cubrir el clítoris. Al encenderse, proporciona una suave succión en el clítoris, mejorando la respuesta sexual femenina al aumentar el flujo sanguíneo tanto en éste órgano como en los genitales externos, resultando en el aumento de la lubricación vaginal y en una mayor capacidad para lograr el orgasmo. Está aprobado por la FDA (Food and Drug Administration) desde el año 2000, para el tratamiento de los trastornos de la excitación y del orgasmo en la mujer(2,5,24-26). Su 
efectividad ha sido documentada, reportando una mejoría de al menos $42 \%$, aunque el número de pacientes en todos los estudios ha sido pequeño $(2,22,27,28)$.

Al considerar todos los aspectos relacionados con la presencia de la anorgasmia femenina, y el hecho de que se dispone de pocas alternativas no farmacológicas; estimulados por el significativo número de mujeres con esta dificultad sexual que solicitan los servicios de la Clínica Sexológica de La Sagrada Familia, en la ciudad de Armenia, Quindío, Colombia, se propuso realizar esta investigación con el objetivo de presentar los resultados de la efectividad y seguridad del uso del dispositivo EROS-CTD en el manejo de la anorgasmia de las mujeres atendidas.

\section{Materiales y métodos}

Se realizó un estudio de reporte de casos, del tipo observacional, descriptivo, prospectivo, de las mujeres intervenidas por trastornos del orgasmo en la Clínica Sexológica de La Sagrada Familia, en la ciudad de Armenia, Quindío, en el periodo comprendido desde el $1^{\circ}$ de febrero de 2012 hasta el 31 de octubre de 2017. El muestreo fue consecutivo.

Los criterios de inclusión fueron: mujeres mayores de 18 años sexualmente activas, atendidas en la Clínica Sexológica por anorgasmia (primaria o secundaria), participación voluntaria en el estudio; los criterios de exclusión fueron embarazo, menopausia, retardo mental, trastornos psiquiátricos y enfermedades vasculares.

El dispositivo EROS se utilizó en todos los encuentros sexuales coitales y durante la masturbación en solitario o en pareja.

El instrumento utilizado para la evaluación de las disfunciones sexuales fue el Índice de Función Sexual Femenina Abreviado-6 (IFSFA-6); este es un cuestionario útil en el diagnóstico de las disfunciones sexuales femeninas, cuenta con una sensibilidad y especificidad $>94 \%$ con un valor de $p<0,001(29)$. El IFSFA- 6 está conformado por 6 preguntas $\mathrm{y}$ ha sido validado en español(30); evalúa el deseo, excitación, lubricación, orgasmo, satisfacción y dolor durante la relación sexual. A cada pregunta se le da una puntuación de 0 al 5, al final el resultado es la suma aritmética de los dominios. Una puntuación igual o inferior a 19 o cuando la puntuación de algún dominio es menor a 3,6 se considera como criterio de riesgo de disfunción sexual, y un valor superior a 19 se relaciona con función sexual femenina normal(29,31).

A las mujeres que cumplían los criterios de inclusión, se les realizó una historia clínica para obtener las variables medidas: sociodemográficas [edad, raza, talla, peso, Índice de masa corporal (IMC), estado civil, ocupación, escolaridad, procedencia, condición espiritual o religiosa)]; variables de comportamiento sexual, como: edad de inicio de vida sexual, actividad sexual (masturbación, sexo oral, coito vaginal o anal), frecuencia promedio semanal de relaciones sexuales, tiempo de convivencia en pareja, antecedente de abuso sexual o violencia sexual en el matrimonio, pareja con disfunción sexual; antecedentes como: hábitos tóxicos (ingesta de alcohol y tabaquismo), consumo de medicamentos, método anticonceptivo, paridad, antecedente de enfermedades orgánicas y quirúrgicos, antecedente personal de depresión, antecedente familiar de trastornos sexuales, antecedente personal de anorgasmia y tratamientos recibidos.

Se indagaron además las preguntas de los dominios de la encuesta IFSFA-6. Cada uno de los resultados de las pacientes fue registrado en un formato especial diseñado por el investigador. La función sexual se evaluó mediante el IFSFA-6, al inicio del estudio luego a los 6 meses, a los 12 meses y a los 24 meses (tiempo en el que se cerró el estudio para cada paciente).

\section{Consideraciones éticas}

Del total de las mujeres que cumplió con los criterios y con indicación terapéutica, se le informó los objetivos de la investigación y el propósito de los resultados del estudio, se les dio la oportunidad de preguntar y aclarar dudas. Todas las pacientes firmaron el consentimiento informado autorizando la participación en la 
investigación, y se garantizó la confidencialidad de la información. A lo largo del proceso se respetó las normas de buena práctica clínica establecidas conforme a las normas éticas del comité de experimentación humana responsable, de acuerdo con la Asociación Médica Mundial y la Declaración de Helsinki(1).

\section{Análisis estadístico}

Los datos fueron analizados en los programas estadísticos SPSS versión 19 y Epi Info versión 3.5.1. Las variables cualitativas fueron expresadas como frecuencias absolutas $y$ relativas (porcentajes). Las variables numéricas se expresaron como media con su desviación estándar (DS).

\section{Resultados}

En el periodo evaluado, de un grupo de 48 pacientes, se incluyeron 45 mujeres $(93,75 \%)$, de las cuales tres $(6,66 \%)$ se negaron a participar. De las 42 mujeres restantes que cumplieron los criterios de selección, $2(4,76 \%)$ no diligenciaron de forma correcta el cuestionario IFSFA- 6 y una $(2,38 \%)$ se retiró antes de completar el cuestionario. De este modo, para el análisis final se tomó en cuenta 39 mujeres (81,25\%), que presentaban trastornos con el orgasmo de acuerdo al IFSFA-6.

La edad media de las mujeres incluidas fue de 35,1 (DS $\pm 3,7$ ) años, en un rango de 18 a 45 años, una mediana de 35 años y un coeficiente de variación de 28\%. La mayoría eran hispanas $(71,79 \%, \quad n=28)$, casadas $(38,46 \%, n=15)$, procedentes del área urbana $(79,48 \% \mathrm{n}=31)$, con educación secundaria $(53,84 \%, \quad n=21)$, empleadas $(61,53 \%, n=24)$ y pertenecientes al régimen de aseguramiento contributivo. El promedio de años de estudios: 10,8 (DS $\pm 4,8$ ) años. El $76,92 \%(n=15)$ ingería alcohol por lo menos una vez a la semana, $48,71 \% \quad(n=19)$ nunca había fumado y el $12,82 \%(n=5)$ fumaba en la actualidad. Respecto al estrato socioeconómico se observó que el 61,53\% (n=24) eran de estrato alto. El 76,92\% (n=27) profesaban la religión católica. La relación multípara a nulípara fue de 2:1. La tabla 1 muestra las características generales y los datos socio-demográficos de las pacientes.

En relación a los antecedentes de salud sexual y reproductiva se encontró una mediana de 3 hijos en la paridad (2 vía cesárea y 1 vía vaginal por mujer), y un rango entre 0 y 6 hijos. La prevalencia global de abortos fue del 30,76\%, de los cuales $17,94 \%$ fueron inducidos y realizados en sitios clandestinos. El 84,61\% de las mujeres utilizaban métodos anticonceptivos con predominio de la anticoncepción hormonal (76,92\%), el $27,27 \%$ tenían como método anticonceptivo el implante subdérmico, la píldora el 24,24\% y la inyección el 21,21\%, mientras que el uso del preservativo solo era utilizado por el $53,84 \%$ de las mujeres solteras.

La edad promedio de inicio de la actividad sexual fue de 16,74 (DS $\pm 1,29$ ) años, el número de parejas sexuales reportó una mediana de 9 , con un rango entre 1 y 15, y la edad de inicio de la masturbación fue de 12,87 (DS $\pm 1,35$ ) años. El promedio de relaciones sexuales semanales arrojó una mediana de 2 , siendo los rangos de frecuencia oscilantes entre $0-4$ encuentros a la semana. La práctica sexual más frecuente es el coito vaginal, y la menos frecuente el coito anal $(35,89 \%)$; la masturbación y el sexo oral son consideradas una práctica común en el $87,17 \%$ y el $89,74 \%$ de las mujeres, respectivamente. El $23,07 \%$ refirió más de 5 años de tiempo de convivencia en pareja. El 38,46\% manifestó que la pareja presentaba alguna disfunción sexual. El $46,15 \%$ refirió que la pareja le había sido infiel y 15,38\% afirmó haber sido infiel. El 79,48\% del total de las mujeres refirió fingir el orgasmo, con frecuencia, debido a la presión de la pareja. Únicamente $10 \quad(25,64 \%)$ mujeres utilizaban juguetes sexuales al inicio del estudio, siendo el vibrador el más utilizado. El 100\% afirmaron ser heterosexuales.

Se encontró que el 7,69\% de las participantes presentaban hipertensión arterial (HTA), el $10,25 \%$ diabetes tipo 2, el 12,82\% dislipidemia; tres de cada diez mujeres recibían terapia para hipotiroidismo. El 15,38\% (n=9) eran histerectomizadas, de las cuales el $85,71 \%$ conservaban ambos ovarios, y al 20,51\% (n=8) 
se les había realizado una cirugía antiincontinencia urinaria. Se observó que el 20,51\% recibía tratamiento para la depresión. El 17,94\% de las pacientes reconoció antecedentes de trastornos sexuales familiares.

El grupo etario de 25 a 34 años (69,23\%) presentó mayores dificultades con el orgasmo, con predominio en las casadas $(73,33 \%)$, procedentes del área rural (75\%), de bajo nivel escolar (88\%) y en las pacientes con antecedentes de histerectomía abdominal $(57,14 \%)$.

El 87,17\% (n=34) expresó que la anorgasmia le afectaba negativamente el desempeño sexual, disminuyendo su capacidad de respuesta a estímulos eróticos; generándoles una vida sexual poco satisfactoria.

En relación con los antecedentes sexuales relevantes, encontramos secuelas de abuso sexual infantil en 9 pacientes $(23,07 \%)$. Se observó incapacidad para comunicarse con la pareja en 8 pacientes $(20,51 \%)$, problemas de violencia con la pareja en 7 pacientes $(17,94 \%)$, temor al abandono en 5 pacientes (12,82\%), temor al embarazo en 4 pacientes $(10,25 \%)$, sentimientos de culpabilidad sexual en 3 pacientes (7,69\%), y factores educativos (desconocimiento funcional y anatómico del cuerpo) en 3 pacientes $(7,69 \%)$.

El $79,48 \%$ de las mujeres refirieron una satisfactoria relación de pareja, y el 43,58\% afirmaron ser mujeres empoderadas de su sexualidad, no teniendo conflictos en discutir con sus parejas las dificultades sexuales. El 41,02\% de las mujeres informó haber sufrido alguna forma de violencia sexual a lo largo de la vida, mientras que el 10,25\% reportó abuso sexual por parte de la pareja.

Las mujeres con dificultades primarias del orgasmo fueron 24 (61,53\%) y 15 con secundarias $(38,36 \%)$. Un total de 18 mujeres (46,15\%), habían consultado con otros profesionales por los trastornos orgásmicos y 21 $(53,84 \%)$ nunca buscaron ayuda profesional previamente. De las mujeres que buscaron ayuda, se encontró que el manejo farmacológico fue el más representativo con 15 casos, para un $83,33 \%$, y entre las opciones el bupropión y apomorfina fueron los más usados. En esta serie no se encontró que alguna de las mujeres tratadas hubiese utilizado juguetes eróticos como parte de la terapia recibida antes del uso del dispositivo EROS.

Tabla 1. Características generales y datos sociodemográficos de las mujeres con anorgasmia tratadas con EROS-CTD

\begin{tabular}{|c|c|}
\hline Variable y categorías & Población n= 39 \\
\hline Edad (años) \pm DS & $35,1 \pm 3,7$ \\
\hline Peso $(\mathrm{Kg}) \pm \mathrm{DS}$ & $62,7 \pm 8,1$ \\
\hline Talla (centímetros) $\pm \mathrm{DS}$ & $158,49 \pm 5,28$ \\
\hline $\mathrm{IMC} \pm \mathrm{DS}$ & $24,6 \pm 4,5$ \\
\hline \multicolumn{2}{|l|}{$\operatorname{Raza} \%$} \\
\hline Hispánicas & 71,79 \\
\hline Afrocolombianas & 28,2 \\
\hline \multicolumn{2}{|l|}{ Estado civil \% } \\
\hline Casadas & 38,46 \\
\hline Unión libre & 25,64 \\
\hline Solteras & 33,33 \\
\hline \multicolumn{2}{|l|}{ Estrato socio-económico \% } \\
\hline Alto & 61,53 \\
\hline Medio & 30,76 \\
\hline Bajo \% & 7,69 \\
\hline \multicolumn{2}{|l|}{ Ocupación \% } \\
\hline Amas de casa & 38,46 \\
\hline Empleadas & 61,53 \\
\hline \multicolumn{2}{|l|}{ Escolaridad \% } \\
\hline Primaria & 10,25 \\
\hline Secundaria & 53,84 \\
\hline Técnica & 20,51 \\
\hline Universitaria & 15,38 \\
\hline \multicolumn{2}{|l|}{ Origen $\%$} \\
\hline Urbano & 79,48 \\
\hline Rural & 20,51 \\
\hline \multicolumn{2}{|l|}{ Religión \% } \\
\hline Católicas & 76,92 \\
\hline Otras & 23,07 \\
\hline
\end{tabular}

En la serie de pacientes tratadas, 12 mujeres $(66,66 \%)$ fueron enfáticas en afirmar que se les realizó potenciación de la erotofilia, ya que expresaron que les habían intentado eliminar las actitudes negativas y prejuicios en torno a la sexualidad. En relación a los tratamientos sexológicos no hubo claridad en el tipo de modelo empleado, sin embargo, ninguno incluyó el entrenamiento en masturbación, pero todas afirmaron haber realizado los ejercicios de Kegel 
por recomendación del terapeuta; lo cual podría estar asociado a que la atención recibida no fue realizada por profesionales en sexología.

Al inicio del estudio, la puntuación del IFSFA-6 en la población global, de las 39 mujeres, fue de 14,37 (DS $\pm 4,7$ ) puntos; siendo el puntaje mayor de 15,57 y el mínimo de 10,75 puntos, con una desviación estándar de $\pm 3,06$ puntos. La puntuación media para el dominio orgasmo fue 1,21 (DS $\pm 0,9$ ).

Al evaluar la puntuación del IFSFA-6, el dominio sexual más alterado es el orgasmo con una puntuación de 1,21 $\pm 0,9$, seguido del deseo
$2,02 \pm 0,7$, siendo el dolor el único dominio con una puntuación superior a 3,6 (Tabla 2). Las mujeres, con anorgasmia primaria, fueron enfáticas en afirmar que nunca habían experimentado orgasmos coitales ni bajo otras circunstancias.

A los seis meses se observó un incremento del dominio orgasmo [2,41 (DS $\pm 0,6)]$, con calificaciones en el IFSFA- 6 de 17,47 (DS $\pm 3,9$ ) puntos. A los doce meses se notó un persistente aumento, con calificaciones en el dominio orgasmo de 3,79 (DS $\pm 0,5$ ), y una puntuación promedio en el IFSFA-6 de 22,21 (DS $\pm 3,6$ ) (Tabla 2).

Tabla 2. Promedios del IFSFA-6 y sus dominios

\begin{tabular}{lrrrr}
\hline & Inicio & 6 meses & 12 meses & 24 meses \\
\hline Dominios & & & & \\
Deseo & $2,02 \pm 0,7$ & $2,44 \pm 0,6$ & $3,51 \pm 0,7$ & $4,13 \pm 0,5$ \\
Excitación & $2,41 \pm 0,9$ & $2,93 \pm 0,8$ & $3,63 \pm 0,6$ & $4,26 \pm 0,4$ \\
Lubricación & $2,45 \pm 0,5$ & $2,95 \pm 0,7$ & $3,62 \pm 0,8$ & $4,31 \pm 0,7$ \\
Orgasmo & $1,21 \pm 0,9$ & $2,41 \pm 0,6$ & $3,79 \pm 0,5$ & $4,22 \pm 0,5$ \\
Satisfacción & $2,61 \pm 0,8$ & $2,96 \pm 0,5$ & $3,85 \pm 0,6$ & $3,45 \pm 0,6$ \\
Dolor & $3,67 \pm 0,9$ & $3,78 \pm 0,7$ & $3,81 \pm 0,4$ & $3,84 \pm 0,8$ \\
Puntaje del IFSFA-6 & $14,37 \pm 4,7$ & $17,47 \pm 3,9$ & $22,21 \pm 3,6$ & $25,21 \pm 3,5$ \\
\hline
\end{tabular}

A los veinticuatro meses se observó un significativo incremento en la puntuación del dominio orgasmo [4,22 (DS $\pm 0,5$ )], con una puntuación promedio en el IFSFA-6 de 25,21 (DS $\pm 3,5$ ). El promedio de seguimiento del uso del dispositivo EROS-CTD, fue 23,7 (DS $\pm 4,5$ ) meses por paciente. En la tabla 2 se describe la puntuación detallada de cada dominio del IFSFA6 al inicio y al final del estudio.

Las mujeres cuyas parejas intervenían, participando en la terapia, fueron las que obtuvieron mejores resultados entre las casadas, estando la puntuación del dominio orgasmo del IFFSA-6, a los 24 meses, en una puntuación media mayor a 4,52 (DS $\pm 1,27)(93,33 \%)$, mientras que en el grupo restante alcanzó una puntuación de 3,65 (DS $\pm 0,68$ ). En aquellas con antecedentes psicológicos (temores, abusos, etc.) se encontró una puntuación media mayor a 3,07 pero inferior a 4,02, correspondiendo al 70,37\%. Por último, en las mujeres con frecuencia sexual igual o mayor de dos encuentros a la semana, la puntuación media fue mayor a 4,54, ubicándolas en los mejores resultados del estudio.

En cuanto a la masturbación, al ingreso del estudio solo el $30,76 \%$ se masturbaban con regularidad (1 - 2 veces por semana). Del total de la población, únicamente el 25,64\% habían usado un juguete erótico, siendo el vibrador el más utilizado; sin embargo, al final del estudio, el $94,87 \%$ de estas mujeres, mantuvo una regularidad de masturbación de 1 - 2 veces por semana, pero el $100 \%$ utilizaban algún juguete sexual, donde el $58,97 \%$ lo usaban en pareja, además que el 74,35\% expresó disfrutar más y mejor la masturbación en pareja. Se reportó una tasa de éxito del $89,74 \%$ para alcanzar el orgasmo con la masturbación en solitario, pero sólo el 78,12\% en pareja (sin inclusión del coito).

Se detectó una diferencia de la respuesta del uso del dispositivo EROS-CTD, durante la masturbación en solitario, entre las mujeres casadas, en unión libre y las solteras (73,3\%, 
$80 \%$ y $84,6 \%$, respectivamente), así como entre las que se masturbaban de larga data y las que no $85,71 \%$ y $83,3 \%$, respectivamente.

La situación orgásmica de las pacientes, luego de finalizado el periodo de los meses de seguimiento es la siguiente: 28 mujeres $(74,35 \%)$ satisfechas con la capacidad de lograron orgasmos con regularidad (4-5/5), 7 $(17,94 \%)$ lograban sus orgasmos con menos regularidad (2-3/5), pero se sentían satisfechas y $4(10,25 \%)$ con dificultades para lograr el orgasmo con facilidad $(<2 / 5)$, ninguna afirmó tener las dificultades o problemas previos al ingreso del estudio. La percepción negativa acerca del dispositivo EROS-CTD fue del 5,12\%.

La tasa de éxito (logro del orgasmo), al final del estudio fue del $89,74 \%(n=35)$ en esta serie de mujeres; al dividir alcanzar el orgasmo entre la masturbación y la pareja (con inclusión del coito), se observan porcentajes de 78,12\% y $89,74 \%$, respectivamente.

Las mujeres con anorgasmia secundaria presentaron tasas bajas de satisfacción $(20,83 \%)$ en relación con las que tenían anorgasmia primaria (53,33\%), y aún menos satisfacción se observó en las que ya habían sido tratadas previamente $(72,22 \%)$, insatisfacción secundaria a tener que recurrir al dispositivo EROS-CTD para lograr del orgasmo.

Durante el seguimiento se percibió que las mujeres mostraron sentimientos de disgusto, molestia y rencor hacia sus parejas en más del 79,48\%, trasladándoles parte de su insatisfacción sexual y anorgasmia a la falta de recursos sexuales. La seguridad del dispositivo EROS-CTD quedó demostrada, ya que ninguna de las mujeres presentó complicaciones relacionadas con el uso del aparato.

\section{Discusión}

La serie de mujeres intervenidas en la consulta sexológica, durante 69 meses, por trastornos del orgasmo, tienen un comportamiento similar en las variables socio-demográficas, tanto en mujeres colombianas como de otros lugares, con respecto a la literatura revisada( ${ }^{(8,32-34)}$.

En esta investigación hubo un bajo porcentaje $(10,25 \%)$ de mujeres insatisfechas con los resultados obtenidos con el uso del dispositivo EROS-CTD, por no tener la oportunidad de lograr orgasmos en todos los encuentros coitales ni con la masturbación; no obstante, se reporta una satisfacción global del $89,74 \%$ con el uso del dispositivo.

En este estudio se observó una alta tasa de éxito a la hora de lograr el orgasmo con la masturbación $(78,12 \%)$, pero esta frecuencia es inferior a la informada por Bronselaer et al., en Bélgica, el cual expresa que el $94 \%$ de las mujeres logró el orgasmo con la estimulación del clítoris(35). La diferencia se podría explicar porque en este estudio las mujeres tenían anorgasmia de base; no obstante, los resultados obtenidos son superiores a los del estudio de Espitia, quien reportó un 47,19\% en poblaciones colombianas $^{(8)}$.

Se encontró mayor frecuencia de disfunción orgásmica en mujeres jóvenes y sexualmente menos experimentadas, siendo concordante con otros estudios donde reportan una tasa de prevalencia del $11 \%$ para el orgasmo inhibido(12); por su parte Guarín-Serrano et al., reportaron que en una población de mujeres universitarias de Bucaramanga, el 24\% nunca había tenido un orgasmo en su vida y el $21 \%$ de las que habían experimentado orgasmos, tenían dificultad para alcanzarlo(34). En el presente estudio, se encontró un mayor porcentaje de anorgasmia primaria $(61,53 \%)$, respecto a la secundaria $(38,36 \%)$.

En la población de estudio se encontró que un $79,48 \%$ fingía el orgasmo como una forma de estar en paz con la pareja, lo cual es superior a lo revisado en la literatura, pues Espitia menciona una frecuencia de simuladoras del orgasmo del $58,34 \%{ }^{(8)}$, mientras en una población de estudiantes universitarias de Estados Unidos, Muehlenhard y Shippee informaron que el 67\% de las mujeres fingen el orgasmo(36); lo cual está asociado a la presión recibida por parte de las 
parejas, tal como lo afirmaron las pacientes del presente estudio.

Al ser la anorgasmia femenina una disfunción sexual que afecta la salud y calidad de vida de las mujeres, dificultando la relación de pareja(2,37,38), llama la atención que en la literatura revisada no sean frecuentes los estudios del manejo no farmacológico, donde el dispositivo EROS-CTD participe dentro de más investigaciones, a pesar de los significativos resultados reportados por Schroder et al., estos en un grupo de mujeres con edad promedio de 43,5 años, a los 3 meses observaron mejoras estadísticamente significativas en todos los dominios del Índice de Función Sexual Femenina (IFSF), aumentando de 17 a 29,4 (puntaje máximo: 36; p<0,001)(39), mientras Billups et al., en la Universidad de Boston, informaron un aumento en la capacidad para alcanzar el orgasmo del $45 \%$ con la terapia EROS-CTD (24),

Respecto a los antecedentes relevantes encontrados, se consideraron relacionados con la anorgasmia en esta serie, el abuso sexual infantil, incapacidad para comunicarse con la pareja y problemas de violencia con la pareja; siendo concordante a lo descrito por IsHa et al., quienes afirman que la prevalencia y las causas de los trastornos del orgasmo en las mujeres son abundantes y multifactoriales(23).

Si el orgasmo es un indicador del ejercicio saludable de la función erótica(2,5) y de la satisfacción sexual, los resultados de esta investigación, concuerdan con la evidencia de una vida sexual poco satisfactoria en la mujer con trastornos del orgasmo.

La diferencia encontrada de la respuesta del uso del dispositivo EROS-CTD, entre las mujeres casadas y las solteras, así como entre las que se masturbaban de larga data y las que no, podría ser explicada por la existencia de la fuerte influencia de la cultura patriarcal y de la tradición de conservadurismo sexual $(2,5)$ de las mujeres participantes de este estudio; lo cual actúa como un factor de aprensión para recurrir a este tipo de prácticas.
La literatura mundial muestra reportes controversiales con relación al resultado del uso del dispositivo EROS-CTD en las mujeres sometidas a tratamiento para la anorgasmia $(27,28,39,40) ; \quad$ sin embargo, en éste estudio se registraron cifras significativamente superiores $(89,74 \%)$. La investigación al analizar el seguimiento y puntuación del IFSFA-6, registró porcentajes de incrementos satisfactorios, probablemente debido a que se trató de mujeres que recibieron asesoría y educación en las bondades del dispositivo, además del involucramiento de la pareja en la terapia. Finalmente, la aceptación reportada por las mujeres para el uso del dispositivo EROS-CTD es significativa, siendo la percepción negativa relativamente baja $(5,12 \%)$, y destacándose el bajo entusiasmo entre mujeres con arraigo espiritual y religioso. Al no presentarse ninguna complicación relacionada con el uso del aparato, se pudo demostrar la seguridad del dispositivo.

Las limitaciones de este estudio se expresan por el pequeño número de participantes, y que no se siguieron todas las mujeres hasta finalizar el estudio. Por otro lado, el hecho de no utilizar una encuesta previamente validada, para evaluar de forma específica la anorgasmia (al ser una variable difícil de medir), se podría alterar la estimación de la prevalencia de la anorgasmia en la población de estudio, dificultándose por ello la extrapolación de los resultados. Entre las fortalezas de esta investigación está que es el primer trabajo de este tipo realizado en Colombia, el tiempo de seguimiento y la oportunidad de seguir contando con estas mujeres para futuros estudios.

\section{Conclusiones}

La efectividad del tratamiento de la anorgasmia con el dispositivo EROS-CTD, en esta serie, ha beneficiado favorablemente a las pacientes, reportando una satisfacción global del 89,74\%; resultado que está por encima de lo descrito en la literatura mundial.

Se hace necesario realizar un control de esta cohorte de mujeres a mayor tiempo y con más participantes para confirmar estos resultados. 


\section{Agradecimientos}

A las mujeres participantes en este estudio, tanto por la disposición en participar en esta investigación, así como por el esfuerzo económico realizado para adquirir el dispositivo.

\section{Conflicto de intereses}

Ninguno declarado por los autores.

\section{Referencias}

1. Laumann EO, Paik A, Rosen RC. Sexual dysfunction in the United States: prevalence and predictors, JAMA. 1999; 281(6):537-44.

2. Espitia-De la Hoz FJ. Sexo Ilimitado/El vuelo hacia una sexualidad más placentera. Primera Edición. Bogotá. Editorial Bolívar. 2015. p.59-65.

3. Kleinplatz PJ. History of the Treatment of Female Sexual Dysfunction(s). Annu Rev Clin Psychol. 2018; 7(14):29-54. doi: 10.1146/annurev-clinpsy-050817084802

4. Sathyanarayana Rao T S, Tandon A, Manohar S, Mathur S. Clinical Practice Guidelines for management of sexual disorders in elderly. Indian J Psychiatry 2018; 60, Suppl S3:397-409. doi: 10.4103/00195545.224478

5. Espitia-De la Hoz FJ. Mi primer año en La Crónica. Primera Edición. Bogotá. Editorial Bolívar. 2016. p.111-112.

6. Rellini AH, Clifton J. Female Orgasmic Disorder. Advances in Psychosomatic Medicine. 2011; 31:35-56. doi:10.1159/000328807

7. Espitia-De la Hoz FJ. Evaluación de la prevalencia de disfunción sexual en mujeres médicos, del Eje Cafetero colombiano, en etapa de climaterio. Arch Med (Manizales) 2017; 17(1):70-7.

8. Espitia-De La Hoz FJ. Prevalence and characterisation of sexual dysfunctions in women, in 12 Colombian cities, 2009-2016. Rev Colomb Obstet Ginecol 2018; 69:9-21. doi: $10.18597 /$ rcog.3035

9. Najafabady MT, Salmani Z, Abedi P. Prevalence and related factors for anorgasmia among reproductive aged women in Hesarak, Iran. Clinics (Sao Paulo). 2011; 66(1):83-86. doi: 10.1590/S180759322011000100015

10. Christensen BS, Grønbaek M, Osler M, Pedersen BV, Graugaard C, Frisch M. Sexual dysfunctions and difficulties in denmark: prevalence and associated sociodemographic factors. Arch Sex Behav. 2011; 40(1):121-32. doi: 10.1007/s10508-010-9599-y

11. Weinberger JM, Houman J, Caron AT, Anger J. Female Sexual Dysfunction: A Systematic Review of Outcomes Across Various Treatment Modalities. Sex Med Rev. 2018. pii: S2050-0521(18)30001-5. doi: 10.1016/j.sxmr.2017.12.004

12. Johnson SD, Phelps DL, Cottler LB. The association of sexual dysfunction and substance use among a community epidemiological sample. Arch Sex Behav.
2004;

33(1):55-63.

doi: 10.1023/B:ASEB.0000007462.97961.5a

13. McCabe MP, Sharlip ID, Lewis R, Atalla E, Balon R, Fisher AD, et al. Incidence and Prevalence of Sexual Dysfunction in Women and Men: A Consensus Statement from the Fourth International Consultation on Sexual Medicine 2015. J Sex Med. 2016; 13(2):14452. doi: 10.1016/j.jsxm.2015.12.034

14. Omani-Samani R, Amini P, Navid B, Sepidarkish M, Maroufizadeh S, Almasi-Hashiani A. Prevalence of Sexual Dysfunction among Infertile Women in Iran: A Systematic Review and Meta-analysis. Int J Fertil Steril. 2019; 12(4):278-283. doi: 10.22074/ijfs.2019.5395

15. Jannini EA, Buisson 0, Rubio-Casillas A. Beyond the Gspot: clitourethrovaginal complex anatomy in female orgasm. Nat Rev Urol. 2014; 11(9):531-8. doi: 10.1038/nrurol.2014.193

16. Puppo V. Anatomy and physiology of the clitoris, vestibular bulbs, and labia minora with a review of the female orgasm and the prevention of female sexual dysfunction. Clin Anat 2013; 26(1):134-152. doi:10.1002/ca.22177

17. Li T, Liao Q, Zhang H, Gao X, Li X, Zhang M. Anatomic Distribution of Nerves and Microvascular Density in the Human Anterior Vaginal Wall: Prospective Study. PLoS One. 2014; 9(11): e110239. doi:10.1371/journal.pone.0110239

18. Yang C, Hakky TS, Carrion R. Evaluation and Treatment of Orgasmic Dysfunction. In: Lipshultz L, Pastuszak A, Goldstein A, Giraldi A, Perelman M. (eds) Management of Sexual Dysfunction in Men and Women. Springer, New York, NY. ISBN: 978-1-49393099-907/978-1-4939-3100-2_15. 2016; 159-170. doi: 10.1007/978-1-4939-3100-2_15

19. Berman JR, Berman L, Werbin T, Goldstein I. Female sexual dysfunction: anatomy, physiology, evaluation and treatment options. Curr Opin Urol. 1999; 9(6):563-8.

20. Krakowsky Y, Grober ED. A practical guide to female sexual dysfunction: An evidence-based review for physicians in Canada. Can Urol Assoc J. 2018; 12(6): 211-16. doi:10.5489/cuaj.4907

21. Laan E, Rellini AH. Can we treat anorgasmia in women? The challenge to experiencing pleasure. Sexual and Relationship Therapy. 2011; 26(4):329-41. doi:10.1080/14681994.2011.649691

22. Cabello-Santamaría F. Disfunciones sexuales femeninas. En Cabello F, Lucas M, editores. Manual Médico de Terapia Sexual. Primera Edición. Madrid: Psimática; 2002. p.281-293.

23. IsHak WW, Bokarius A, Jeffrey JK, Davis MC Bakhta Y. Disorders of Orgasm in Women: A Literature Review of Etiology and Current Treatments. The Journal of Sexual Medicine. 2010; 7(10):3254-68. doi:10.1111/j.1743-6109.2010.01928.x

24. Billups KL, Berman L, Berman J, Metz ME, Glennon ME, Goldstein I. A new non-pharmacological vacuum therapy for female sexual dysfunction. J Sex Marital Ther. 2001; 27(5):435-41. 
25. Schroder M, Bieber EJ, Mell LK, Mundt AJ. Eros therapy: Treatment of sexual dysfunction in postmenopausal women not on hormone replacement. Obstet Gynecol. 2003; 101(4):95S.

26. Wilson SK, Delk JR 2nd, Billups KL. Treating symptoms of female sexual arousal disorder with the ErosClitoral Therapy Device. J Gend Specif Med. 2001; 4(2):54-8.

27. Billups K, Bakdash D, Savage M, Flaherty E, Goldstein I. Vacuum-induced clitoral engorgement for treatment of female sexual dysfunction in diabetic women. J Urol 2002; 167(4):281 (abst 1105).

28. Billups KL. The role of mechanical devices in treating female sexual dysfunction and enhancing the female sexual response. World J Urol. 2002; 20(2):137-41. doi: $10.1007 / \mathrm{s} 00345-002-0269-0$

29. Isidori AM, Pozza C, Esposito K, Giugliano D, Morano S, Vignozzi L, et al. Development and validation of a 6item version of the female sexual function index (FSFI) as a diagnostic tool for female sexual dysfunction. J Sex Med. 2010; 7(3):1139-46. doi: 10.1111/j.17436109.2009.01635.x

30. Chedraui P, Pérez-López FR, Sánchez H, Aguirre W, Martínez N, Miranda O. Assessment of sexual function of mid-aged Ecuadorian women with the 6-item Female Sexual Function Index. Maturitas. 2012; 71(4):407-12. doi: 10.1016/j.maturitas.2012.01.013

31. Carpenter JS, Jones SM, Studts CR, Heiman JR, Reed SD, Newton KM. Female Sexual Function Index Short Version: A MsFLASH Item Response Analysis. Arch Sex Behav. 2016; 45(8):1897-1905. doi: 10.1007/s10508016-0804-5

32. Espitia-De La Hoz, FJ. Prevalencia de disfunción sexual en mujeres del Eje Cafetero. Rev.cienc.biomed 2016; $7(1): 25-33$.

33. Rivera GP, González IR, Vargas Peña ML. Disfunciones sexuales en la mujer mapuche. Rev Chil Urol. 2004; 69:68-72.

34. Guarín-Serrano R, Cadena-Afanador L, MujicaRodríguez AM, Ochoa-Vera ME, Useche-Aldana B. Prevalencia de orgasmo en mujeres universitarias de Bucaramanga (Colombia), 2013. Rev Colomb Obstet Ginecol. 2014; 65(4):330-37. doi: 10.18597/rcog.37

35. Bronselaer G, Callens N, De Sutter P, De Cuypere G, T'Sjoen G, Cools, et al. Self-Assessment of Genital Anatomy and Sexual Function within a Belgian, Dutch-Speaking Female Population: A Validation Study. The Journal of Sexual Medicine. 2013; 10(12), 3006-18. doi:10.1111/jsm.12326

36. Muehlenhard CL, Shippee SK. Men's and women's reports of pretending orgasm. J Sex Res. 2010; 47(6):552-67. doi: 10.1080/00224490903171794

37. Hurlbert DF, Apt C. The coital alignment technique and directed masturbation: a comparative study on female orgasm. J Sex Marital Ther. 1995 Spring; 21(1):21-9. doi: 10.1080/00926239508405968

38. Hisasue S, Kumamoto Y, Sato Y, Masumori N, Horita H, Kato $\mathrm{R}$, et al. Prevalence of female sexual dysfunction symptoms and its relationship to quality of life: a Japanese female cohort study. Urology. 2005; 65(1):143-8. doi: 10.1016/j.urology.2004.08.003
39. Schroder M, Mell LK, Hurteau J.A, Collins YC, Rotmensch J, Waggoner S E. Clitoral therapy device for treatment of sexual dysfunction in irradiated cervical cancer patients. International Journal of Radiation Oncology Biology Physics. 2005; 61(4):1078-86. doi:10.1016/j.ijrobp.2004.07.728

40. Munarriz R, Maitland S, Garcia SP, Talakoub L, Goldstein I. A prospective duplex Doppler ultrasonographic study in women with sexual arousal disorder to objectively assess genital engorgement induced by EROS therapy. J Sex Marital Ther. 2003; 29 Suppl 1:85-94. doi: 10.1080/713847133 